\title{
What we don't know about osteoporosis
}

\author{
E. M. Lewiecki ${ }^{1} \cdot$ N. Binkley ${ }^{2}$
}

Received: 25 January 2016 / Accepted: 3 February 2016 / Published online: 22 February 2016

(C) Italian Society of Endocrinology (SIE) 2016

It ain't what you don't know that gets you into trouble.

It's what you know for sure that just ain't so.

Mark Twain, American humorist, 1835-1910.

In science as in politics, there is no shortage of pundits expounding on why things are the way they are and what we should do to make things better. In hindsight, many of them have been wrong. The field of osteoporosis is strewn with discarded theories, blind alleys, and false leads. Even the great Fuller Albright, who has been called the father of modern endocrinology, was mistaken in some of his ideas about osteoporosis. He recognized that postmenopausal osteoporosis was a disease caused by estrogen deficiency, but incorrectly attributed this to impaired bone formation [1]. The first report of bone mass increases with daily parathyroid hormone injections was published in 1929 and validated a few years later, yet not pursued as a potential treatment for osteoporosis until 40 years later [2]. At one time, but no longer, sodium fluoride was a promising agent for osteoporosis treatment. More recently, it was felt that postmenopausal women at low fracture risk should receive bisphosphonates, a concept that may have merit for some, but is no longer in vogue.

The following are a few examples of current osteoporosis concepts that may, or may not, be found correct by those who follow us, and limitations in our knowledge that may be impeding our progress in treating this disease.

E. M. Lewiecki

mlewiecki@gmail.com

1 New Mexico Clinical Research \& Osteoporosis Center, 300 Oak Street NE, Albuquerque, NM 87106, USA

2 University of Wisconsin Osteoporosis Clinical Center and Research Program, Madison, WI, USA
Bone remodeling Our current understanding is that postmenopausal osteoporosis results from a bone remodeling imbalance with bone resorption exceeding bone formation and that the medications approved for osteoporosis treatment all work by modulating bone remodeling in a favorable way. This is almost certainly correct, yet there is much more to be learned about bone remodeling that might enhance our understanding of the drugs we now use, improve their safety, and assist in developing new ones. A vast gap remains in our understanding of bone remodeling at the cellular level. There is much to learn about the molecular regulators that determine differentiation, activity, and survival of osteoclasts, osteoblasts, and osteocytes. As knowledge of cellular and molecular mediators of "crosstalk" between bone and muscle advances, new approaches to reducing fracture risk may emerge.

In addition to enhanced understanding on the cellular level, we must learn how to best modulate remodeling and for how long to continue an intervention. Bisphosphonates are unique among osteoporosis medications in having a long skeletal retention time with persistence of antiresorptive effect (for an uncertain, and almost certainly variable, duration of time) after discontinuation. This has resulted in the concept of bisphosphonate "holidays" where patients discontinue treatment, hopefully continuing to benefit from anti-fracture efficacy for an undefined length of time while having an improved safety profile. Given the paucity of data supporting bisphosphonate holidays, uncertainty remains on when, if ever, and for whom, to start a holiday, when to end it, and how to monitor patients during a holiday. It is not clear how best to treat a patient who has been on longterm bisphosphonate therapy (e.g., 10 years or more) and remains at high risk for fracture, particularly if a full course of treatment with teriparatide has previously been administered. Finally, enhanced understanding of bone remodeling 
may allow us to identify individuals who may be at risk for rare adverse events, such as osteonecrosis of the jaw (ONJ) and atypical femur fracture (AFF), that are associated with some antiresorptive agents.

Calcium and vitamin D Calcium and vitamin D adequacy is a fundamental component of osteoporosis treatment, yet the use of these essential skeletal nutrients is controversial. There is vigorous debate and conflicting data on whether calcium supplementation is, or is not, associated with adverse cardiovascular effects. Similarly, there are strong differences of opinion on the optimal dose for vitamin $\mathrm{D}$ supplementation, target serum $25(\mathrm{OH}) \mathrm{D}$ concentrations, the role of vitamin $\mathrm{D}$ in calcium absorption and in falls risk, the skeletal and non-skeletal effects of vitamin $\mathrm{D}$, and the best approach to defining vitamin $\mathrm{D}$ inadequacy $[3,4]$. Some, perhaps much, of this controversy is due to flaws in clinical trial design that cannot be overcome with meta-analyses. Indeed, some have called for a moratorium on calcium and vitamin D meta-analyses. We can expect these controversies to continue until there are advances on many research fronts. Considering our knowledge limitations regarding major nutritional factors (calcium/vitamin D), it is hardly surprising that even less is known about other nutrients with potential skeletal effects, such as magnesium, vitamin $\mathrm{A}$, and vitamin $\mathrm{K}$.

Medications Current osteoporosis medications are categorized according to their effects on bone remodeling. Those that act by decreasing bone remodeling (e.g., bisphosphonates, denosumab) are called antiresorptive agents, while those that act by increasing bone remodeling (e.g., teriparatide) are called anabolic. However, there are differences in the skeletal response to some drugs in the same category that are not easily explained. For example, the continuing BMD increase with 10 years of continuous denosumab treatment is quite different than the pattern of response observed with long-term bisphosphonates, possibly due to a "permissive" effect of denosumab on bone modeling. Perhaps a better understanding of the mechanisms responsible for such large increases in BMD could ultimately lead to a "cure" of osteoporosis. In addition, more medication options are sorely needed. Some drugs currently in development (e.g., odanacatib, romosozumab) do not fit the current antiresorptive/anabolic dichotomy due to differential effects on bone resorption and formation. An updated system of drug classification may emerge as we come to better understand the mechanisms of action of these and other future medications. As the basic science of bone biology advances, more new compounds with novel mechanisms of action are likely to be developed, provided there are no overwhelming financial, political, or regulatory obstacles. Finally, just as multi-drug therapy is necessary for many chronic diseases; it seems likely that this is also true for osteoporosis, but combination therapy is rarely used today. Concepts on combining (sequentially or simultaneously) and cycling drugs for osteoporosis seem likely to evolve as more data become available.

Osteonecrosis of the jaw and atypical femur fracture $\mathrm{ONJ}$ and AFF are associated with bisphosphonates and denosumab [5, 6], most often in cancer patients receiving doses far higher than used for osteoporosis. The causality and pathophysiology of these rare events are not well understood. An example of a blind alley in the quest to reduce ONJ risk was the notion that a low serum level of the bone resorption marker collagen type $1 \mathrm{C}$-telopeptide (CTX) predicted high ONJ risk with dental surgery, an idea that was subsequently not supported by data [7]. Although fear of ONJ and AFFs has caused great angst with many patients and some physicians, progress in preventing or mitigating ONJ and AFFs will remain limited until we have a better understanding of their origins.

Treatment gap Medications do not work if patients do not take them. Unfortunately, the osteoporosis treatment gap (the difference between the number or percentage of patients who could benefit from osteoporosis treatment and those who take it) is large and appears to be getting worse, even following hip fracture [8]. The origins of this treatment gap are multifactorial, but in part relate to fear of rare adverse events, such as ONJ and AFF. This fear is fueled by well intended but often unbalanced media reports of complex medical issues [9]. Many patients, and some physicians, do not fully understand the potentially serious quality of life consequences of fractures and the balance of expected benefits and possible risks with treatment. Uncertainty regarding duration of therapy with bisphosphonates is leading to some high risk patients stopping therapy, on their own or on the advice of their physicians, when the benefits probably still outweigh the risks. More study is needed to develop effective clinical tools for risk benefit communication. This may serve to reduce the treatment gap and improve long-term adherence to therapy.

Clinical practice guidelines All guidelines are wrong, but good ones may be useful. Take, for example, the US National Osteoporosis Foundation (NOF) recommendations for osteoporosis treatment [10]. These are based on a model of annual US age-specific incidence of clinical fractures, with many assumptions about drug costs, adherence to therapy, effectiveness, and societal willingnessto-pay. An analysis concluded that osteoporosis treatment was cost-effective when the 10-year hip fracture probability using the US version of FRAX was approximately $3 \%$. Although this guideline represents an advance in the care of osteoporosis in the US, the assumptions upon which it was based were in part arbitrary and certainly are no longer correct, if they ever were. In some other countries, such as the UK, FRAX is used differently, often without BMD testing, with a fracture risk treatment threshold that 
varies by age [11]. Sometimes it is the cost of drug therapy rather than a fracture risk intervention threshold that drives treatment decisions. The optimal approach for determining intervention thresholds is not clear, but may appropriately vary according to local healthcare resources and priorities.

Defining osteoporosis Is osteoporosis a disease, a disorder, or one of many risk factors for fracture? Should it be diagnosed according to BMD, by level of fracture risk, or something else? Should conditions that increase fracture risk independently of BMD (e.g., chronic glucocorticoid therapy, type 2 diabetes mellitus, monoclonal gammopathy) play a role in diagnosing osteoporosis? For many years the osteoporosis field has focused upon the integrity of bone and its association with fractures; since 1994, a BMD T-score $\leq-2.5$ measured by DXA at specific skeletal sites has been used to diagnose osteoporosis in clinical practice. However, the majority of fragility fractures occur in individuals with a T-score better than -2.5 and many result from falling. As such, there is increasing recognition of role played by falls risk and sarcopenia (the age-related loss of muscle mass and strength) in fracture occurrence. Attempts to reach a consensus for defining and diagnosing sarcopenia are ongoing. Similarly, the criteria for defining and diagnosing osteoporosis may continue to evolve in the future. Medications to improve muscle function in older adults are currently being evaluated in phase $2 / 3$ clinical trials. As the importance of sarcopenia as a fracture risk factor becomes more widely recognized, and medications that improve muscle function are developed, greater attention may be focused on fragility fracture rather than osteoporosis as the disease. Indeed, osteoporosis, sarcopenia, diabetes, obesity, and other conditions may come to be recognized as components of a syndrome leading to elevated fracture risk.

We should not condemn those who have traveled down the blind alleys of research, but applaud them. After all, it is often through mistakes, sometimes long forgotten, that great advances are made. Researchers and clinicians who are able to recognize mistakes, learn from them, and persevere when others have given up, are often those who make the greatest discoveries. We propose blending our passion with humility, appreciating the many things that we do not know and recognizing that what we say with certainty today may be the folly of the future. Great progress has been made in managing osteoporosis over the past two decades, but there remains much more to learn.

Since some of today's truths may turn out to be tomorrow's fiction, it behooves osteoporosis researchers and clinicians to think critically and not blindly accept conventional wisdom and the apparent truths of clinical trials and meta-analyses. We must all continue to learn from advances in understanding the pathophysiology of osteoporosis and consider how our interventions favorably, or unfavorably alter it. Experiences in the past cause one to wonder whether there is already evidence for effective osteoporosis treatments that should be pursued but are not, or evidence of harm with treatments that we are now using that is deserving of more attention.

\section{Compliance with ethical standards}

Conflict of interest EML has received institutional grant/research support from Amgen, Merck, and Eli Lilly; he has served on scientific advisory boards for Amgen, Merck, Eli Lilly, Radius Health, AgNovos Healthcare, Alexion, Shire, and AbbVie. NB has received institutional grant/research support from Amgen, Merck, Eli Lilly and GE Healthcare; he has served on scientific advisory boards for Amgen, Merck and Eli Lilly.

Ethical approval This article does not contain any studies with human participants or animals performed by any of the authors.

Informed consent No informed consent.

\section{References}

1. Byreddy DV, Bouchonville MF II, Lewiecki EM (2015) Druginduced osteoporosis: from Fuller Albright to aromatase inhibitors. Climacteric 18(Suppl 2):39-46

2. Potts JT (2005) Parathyroid hormone: past and present. J Endocrinol 187:311-325

3. Heaney RP (2014) Toward a physiological referent for the vitamin D requirement. J Endocrinol Invest 37:1127-1130

4. Reid IR (2015) Effects of vitamin D supplements on bone density. J Endocrinol Invest 38:91-94

5. Khan AA, Morrison A, Hanley DA et al (2015) Diagnosis and management of osteonecrosis of the jaw: a systematic review and international consensus. J Bone Miner Res 30:3-23

6. Shane E, Burr D, Abrahamsen B et al (2014) Atypical subtrochanteric and diaphyseal femoral fractures: second report of a task force of the American Society for Bone and Mineral Research. J Bone Miner Res 29:1-23

7. Baim S, Miller PD (2009) Assessing the clinical utility of serum CTX in postmenopausal osteoporosis and its use in predicting risk of osteonecrosis of the jaw. J Bone Miner Res 24:561-574

8. Solomon DH, Johnston SS, Boytsov NN, McMorrow D, Lane JM, Krohn KD (2014) Osteoporosis medication use after hip fracture in U.S. patients between 2002 and 2011. J Bone Miner Res 29:1929-1937

9. Rothman MS, Miller PD, Lewiecki EM, Bilezikian JP (2014) Bone density testing: science, the media, and patient care. Curr Osteoporos Rep 12:227-229

10. Cosman F, de Beur SJ, LeBoff MS, Lewiecki EM, Tanner B, Randall S, Lindsay R (2014) Clinician's guide to prevention and treatment of osteoporosis. Osteoporos Int 25:2359-2381

11. Compston J, Bowring C, Cooper A et al (2013) Diagnosis and management of osteoporosis in postmenopausal women and older men in the UK: National Osteoporosis Guideline Group (NOGG) update 2013. Maturitas 75:392-396 